\title{
Acousto-optic signal processors for transmission and reception of phased-array antenna signals
}

\author{
Nabeel A. Riza and Demetri Psaltis
}

\begin{abstract}
Novel acousto-optic processors for control and signal processing in phased-array antennas are presented. These processors can operate in both the antenna transmit and receive modes. An experimental acoustooptic processor is demonstrated in the laboratory. This optical technique replaces all the phase-shifting devices required in electronically controlled phased-array antennas.

Key words: Phased-array antenna, acousto-optic device, signal phase.
\end{abstract}

\section{Introduction}

The application of optical processing for phased-array antennas was introduced by Lambert et al. in the 1960's. ${ }^{1}$ They proposed a signal-processing technique for extracting angle/Doppler target information from multiple target-return signals provided by the phasedarray antenna. ${ }^{2}$ This approach was limited to processing to received antenna signals, and it could not be used to generate drive signals to provide desired antenna beam shape and direction. Subsequent research in optical phased-array antenna processing revolved around the Lambert processor, and most efforts used better hardware to get improved experimental results. ${ }^{3,4}$ Recently there has been interest in using optical techniques for signal generation and control of phased-array antennas. ${ }^{5}$ Some researchers have proposed the use of optical fibers for time-delay beam steering, while others have suggested interferometric optical architectures for phase-based antenna beam formation. ${ }^{6-10}$ In this paper we introduce an in-line additive acousto-optic technique for signal generation and reception in phased-array antennas operating in the lower half of the radar frequency spectrum, that is, at $10 \mathrm{GHz}$ and below. ${ }^{11,12}$ The techniques are based on

N. A. Riza is with the General Electric Corporate Research and Development Center, Building KW, Room B617, Schenectady, New York 12301. D. Psaltis is with the Department of Electrical Engineering, California Institute of Technology, Pasadena, California 91125.

Received 2 November 1990.

0003-6935/91/233294-10\$05.00/0.

(C) 1991 Optical Society of America. the phase beam-steering principle and eliminate all the microwave phase shifters and power dividers required in typical electronically controlled phased-array antennas. The optical system has a simple and mechanically stable design. A single control signal is required for positioning the antenna beam at a desired scan angle. In addition, this acousto-optic (AO) technique can be adapted to provide multiple beams. ${ }^{13}$ Finally, the technique is applicable for signal transmission as well as reception, thus making a reversible processor.

We begin with an introduction to phased-array antenna theory. The AO phased-array antenna signal processors are introduced along with an analytical description of processor operating principles. A processor is built with tellurium dioxide acousto-optic devices (AOD's). The test processor is built for an antenna carrier frequency of $120 \mathrm{MHz}$. A number of test experiments, such as frequency scan linearity, and $0-2 \pi$ phase shift of the carrier with control frequency change, are performed on the processors. Data from the processor are compared with the ideal performance predicted by system analysis. Next, the processor principles involved in analyzing received antenna signals are described, and the features of the optical techniques are highlighted. In conclusion, we describe why this optical approach to phased array antenna signal processing can provide a great reduction in system complexity, and simplicity in operation and control, compared with electronic phase-shifter modulebased array antennas.

\section{Phased-Array Antennas}

An array antenna consists of a number of individual radiating olomonts. The relative amplitude and phase 


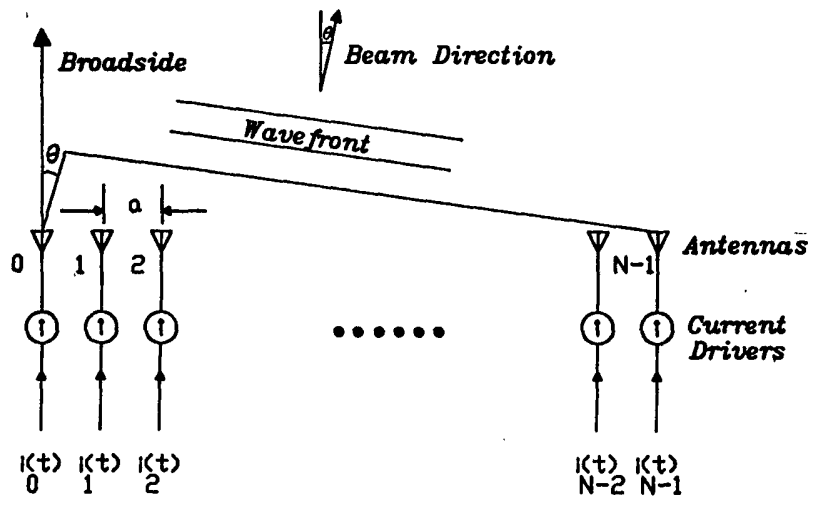

Antenna Signals with Appropriate Phases

Fig. 1. Linear phased-array antenna configuration.

of the currents driving the individual antenna elements are controlled to generate an antenna beam pattern resulting from the superposition of all the radiating elements. A linear array consists of antenna elements arranged along a straight line. This type of array can be used to provide broad coverage in one direction and narrow beamwidth in the orthogonal direction. We shall consider a linear array of $N$ elements equally spaced a distance $a$ apart, with each element being equivalent to an isotropic point source (sink) radiating (receiving) uniformly in all directions. The array antenna shown in Fig. 1 is a transmitting antenna, although, by the reciprocity principle of electromagnetics, the same array can be considered a receiving antenna. To transmit energy at an angle $\theta$ with respect to the array normal direction, we require that the phase difference of signals driving adjacent elements be ${ }^{14}$

$$
\alpha=2 \pi(a / \lambda) \sin \theta,
$$

where $\lambda$ is the radiated wavelength. Thus adjacent antenna elements in the array are driven by signals that satisfy the phase condition in Eq. (1). The amplitudes of these currents can be suitably selected to yield desired beam shapes. For simplicity of analysis, we consider the amplitudes to be all the same and equal to unity. Element 0 in Fig. 1 is taken as the reference antenna with zero phase. Each individual antenna radiates its own pattern, and the combined far-field radiation observed at an angle $\theta$ that is due to all the elements in the array is approximated as the sum

$$
E \approx \sum_{n=0}^{N-1} \sin (\omega t+n \alpha)=\sin [\omega t+(N-1) \alpha / 2] \frac{\sin (N \alpha / 2)}{\sin (\alpha / 2)},
$$

where $\omega$ is the angular frequency of the antenna. The first term is the antenna carrier signal with a phase $(N$ $-1) \alpha / 2$. This phase can be set to zero if the phase reference is taken at the center of the array. The second term is the radiation pattern. When the main beam is pointing in the direction $\theta=\theta_{0}$, the normalized radiation intensity pattern can be expressed as

$$
G(\theta)=\frac{\sin ^{2}\left[N \pi(a / \lambda)\left(\sin \theta-\sin \theta_{0}\right)\right]}{N^{2} \sin ^{2}\left[\pi(a / \lambda)\left(\sin \psi-\sin \theta_{0}\right)\right]},
$$

which is maximum when $\sin \theta=\sin \theta_{0}$. The main beam can be rotated by varying the phase difference $\alpha$ $=2 \pi(a / \lambda) \sin \theta_{0}$ of the signals driving the array antennas. Note that to prevent grating lobes from appearing over the antenna beam scan width, one must make the antenna element spacing of the order of half of the carrier wavelength. In addition, unlike for true timedelay beam steering, with phase steering the antenna's instantaneous bandwidth is limited to a small fraction of the carrier signal to prevent beam squinting.

\section{Acousto-Optic Phased-Array Antenna Signal Processors}

Frequency-controlled beam steerer 1, shown in Fig. 2, is an optical phased-array antenna signal processor applicable for generating the necessary signals for beam formation and beam scanning in linear antenna arrays. The processor consists of two AOD's, a highspeed detector array, a coherent light source, imaging and Fourier-transforming optics, and two signal generators to provide the antenna carrier and control frequency signals. The signals $s_{1}(t)$ and $s_{2}(t)$ are fed into AOD1 and AOD2, respectively, where

$$
s_{1}(t)=a \cos \omega_{c} t, \quad s_{2}(t)=a \cos \left[\left(\omega_{c}+\omega_{0}\right) t\right],
$$

$f_{c}=\omega_{c} / 2 \pi$ is the center frequency of the AOD's, and $\omega_{0} /$ $2 \pi$ is the control frequency for steering the array antenna beam. Light from the laser, after being collimated along the $x$ direction and focused along the $y$ direction, is made incident at the Bragg angle in AOD1. The dc and +1 diffracted order light from AOD1 is 1:1 imaged onto the acoustic column in AOD2 such that the dc light from AOD1 is Bragg matched to that of AOD2. This results in a -1-order diffracted light from AOD2, while the +1-order light from AOD1 passes through AOD2 essentially unaffected. These almost collinear +1- and -1-order diffracted beams from AOD1 and AOD2, respectively, are imaged onto a detector array with magnification $M$. The dc light is blocked in the Fourier plane of AOD2, and the resulting optical field incident upon the detector plane is given by

\section{ACOUSTO-OPTIC PHASED ARRAY RADAR PROCESSOR}
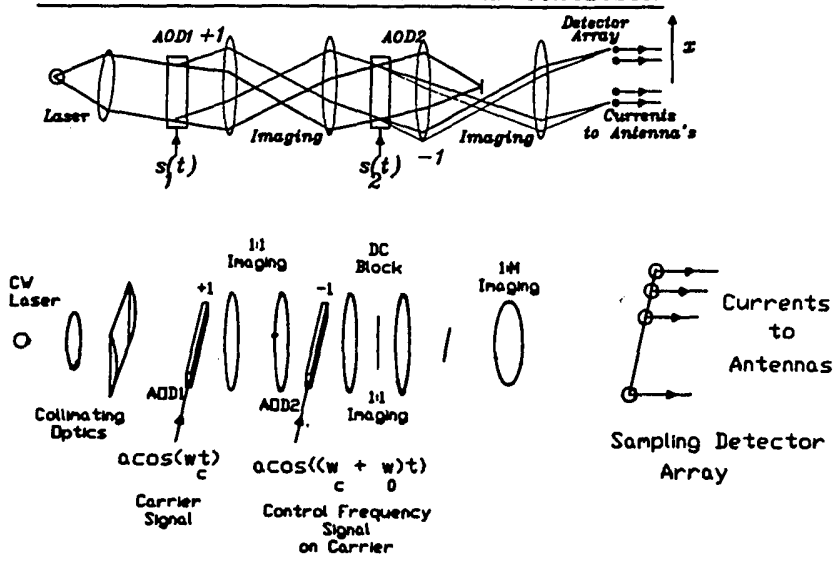

Fig. 2. Frequency-controlled beam steerer 1 . 


$$
\begin{aligned}
E(x, t) & =\left[\exp \left(-j 2 x \frac{\sin \theta_{a}}{\lambda} x\right) \tilde{s}_{1}\left(t-\frac{x}{M \nu_{a}}\right)\right. \\
& \left.+\exp \left(j 2 x \frac{\sin \theta_{a}}{\lambda} x\right) \tilde{s}_{2}{ }^{*}\left(t+\frac{x}{M \nu_{a}}\right)\right] \operatorname{rect}\left(\frac{x-0.5 M X}{M X}\right),
\end{aligned}
$$

where $\left(\sin \theta_{B}\right) / \lambda=f_{c} / 2 \nu_{a}, \nu_{a}$ is the acoustic signal velocity in the AOD's and $\tilde{s}_{1}$ and $\tilde{s}_{2}{ }^{*}$ are the +1 and -1 diffracted signal representations, respectively, given by

$$
\tilde{s}_{1}=\frac{1}{2} a \exp \left(-j \omega_{\mathrm{c}}\right), \quad \tilde{s}_{2}{ }^{*}=\frac{1}{2} a \exp \left[j\left(\omega_{\mathrm{c}}+\omega_{0}\right) t\right] .
$$

Using the expressions for the signals in Eqs. (6), we can write the intensity at the detector plane as

$$
\begin{array}{r}
I(x, t) \propto|E(x, t)|^{2}=\frac{a^{2}}{2}+\cos \left[\left(2 \omega_{c}+\omega_{0}\right) t-\frac{\omega_{0}}{M \nu_{a}} x\right] \\
\times \operatorname{rect}\left(\frac{x-0.5 M X}{M X}\right),
\end{array}
$$

where $a^{2} / 2=$ constant bias and $G_{0}=a^{2} / 2$. The expression in relation (7) consists of a uniform bias term and a signal term with both temporal and spatial modulation. Recall from our discussion on phased-array beam steering that phase-based beam steering requires a set of antenna drive signals on the antenna temporal carrier with appropriate phase values. The expression in relation (7) shows that we can obtain this set of antenna drive signals by simply using a detector array to sample appropriately the phase distribution of the temporally varying intensity pattern. In the case of uniform linear phase sampling, the detectors are placed at an equal distance $d$ from one another. The signal generated at the $n$th detector after spatial integration over the detector's photosensitive area $d_{x} \times d_{y}$ is amplified by a high-frequency amplifier. If the spatial period of the intensity pattern is much greater than the size of the detector in the sampling direction (that is, $u_{x} d_{x} \ll 1$, where $u_{x}=f_{0} / M \nu_{a}$ is the spatial frequency generated along the detector array and $d_{x}$ is the width of the detector along the sampling $x$ direction), then the current produced by the $n$th detector can be approximated as (see Subsection V.D below)

$$
i_{n}(t) \approx G \cos \left[\left(2 \omega_{c}+\omega_{0}\right) t-n \frac{\omega_{0} d}{M \nu_{a}}\right]
$$

where the bias term has been dropped, $G=0.5 G_{0} d_{x} d_{y}$, $n=0,1,2, \ldots, N-1$, and $N$ is the total number of detectors used in the linear sampling array. Note that approximation (8) gives the drive current fed to the $n$th antenna in a uniform linear phased array for achieving beam steering. This current can be expressed as

$$
i_{n}(t)=G \cos (\omega t-n \varphi)
$$

where the antenna carrier angular frequency is

$$
\omega=2 \omega_{c}+\omega_{0}
$$

and the phase difference between signals in adjacent antennas in the array is

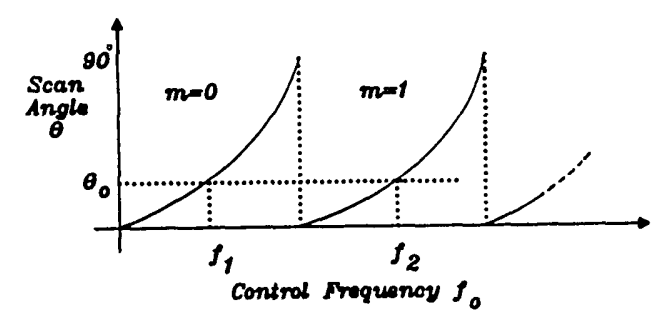

Fig. 3. Antenna beam-scan angle versus processor control frequency behavior.

$$
\varphi=\frac{\omega_{0} d}{M \nu_{a}} .
$$

From phased-array antenna theory, recall that to position the antenna beam at an angle $\theta=\theta_{0}$, we would require that

$$
\varphi=\alpha+2 m \pi=2 \pi(\alpha / \lambda) \sin \theta_{0}+m 2 \pi,
$$

where $m=0, \pm 1, \pm 2, \ldots$ Substituting the value $\varphi$ into Eq. (12) we get

$$
2 \pi(a / \lambda) \sin \theta_{0}=\frac{\omega_{0} d}{M v_{a}}-m 2 \pi, \quad \sin \theta_{0}=(a / \lambda)\left(\frac{f_{0} d}{M v_{a}}-m\right) .
$$

If Eqs. (13) are used, the design equation for the optical processor to achieve a beam position $\theta_{0}$ for a control frequency $f_{0}$ is

$$
\theta_{0}=\sin ^{-1}\left[(a / \lambda)\left(\frac{f_{0} d}{M \nu_{a}}-m\right)\right]
$$

This equation can alternatively be written as

$$
f_{0}=\left[(a / \lambda) \sin \theta_{0}+m\right]\left(\frac{M \nu_{a}}{d}\right) \text {. }
$$

Figure 3 shows graphically the relationship between the antenna beam-scan angle and the processor control frequency. Note that the same angle $\theta_{0}$ can be obtained for multiple values of the frequency $f_{0}$ corresponding to different values of $m$. This permits the generation of multiple simultaneous beams; creating many applications such as multitarget tracking and secured transmission. Also note that, since we are using the phase-shift beam-steering technique, accurate beam pointing requires the antenna instantaneous microwave bandwidth to be $<2 \%$ of the antenna carrier frequency. ${ }^{15}$ This implies that

$$
\frac{\Delta f_{0}}{f^{\prime}} \ll 1,
$$

where $\Delta f_{0}$ is the maximum control frequency change required for the maximum beam-scan angle and $f^{\prime}$ is the antenna carrier center frequency, where $f^{\prime}=2 f_{c}+$ $\Delta f_{0} / 2$.

An alternative design, that for frequency-controlled beam steerer 2, is shown in Fig. 4; in this design the transmitted antenna carrier frequency $f^{\prime}$ is independent of the control frequency $f_{0}$. The optical apparatus of this signal processor is similar to that of the signal processor in Fig. 2, except that different electronic signals are used. Two oscillators are required for this system. One oscillator is a low-frequency sig- 


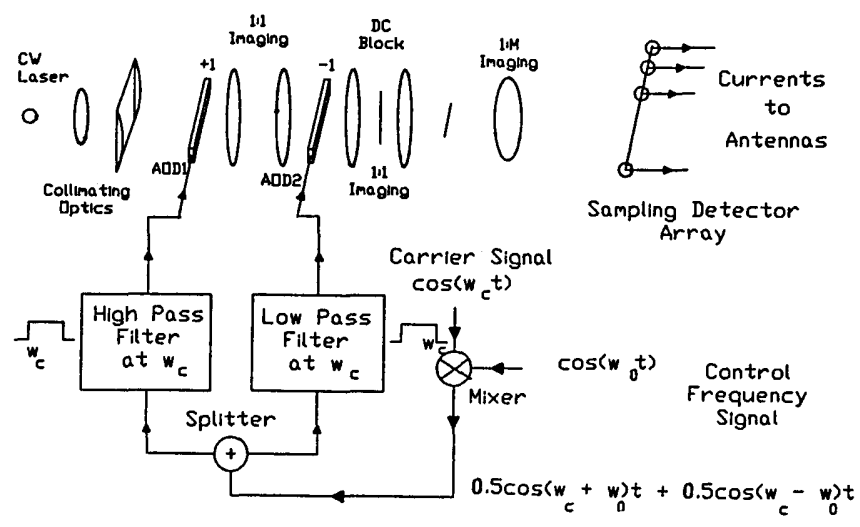

Fig. 4. Frequency-controlled beam steerer 2 .

nal generator providing the scan control frequency $f_{0}$, while the other is a very-high-frequency oscillator operating at half of the desired antenna carrier frequency (also equal to the AOD center frequency). The two signals are mixed together in a standard microwave mixer, giving

$$
s(t)=2 a \cos \omega_{0} t \cos \omega_{c} t=a \cos \left(\omega_{c}+\omega_{0}\right) t+a \cos \left(\omega_{c}-\omega_{0}\right) t .
$$

This signal is split by a microwave power divider into two paths. One path goes through an upper sideband filter at frequency $\omega_{c}$, giving the signal $s_{1}(t)=a \cos \left(\omega_{c}\right.$ $\left.+\omega_{0}\right) t$ that is fed to AOD1. The other path goes to a lower sideband filter at frequency $\omega_{c}$, giving the signal $s_{2}(t)=a \cos \left(\omega_{c}-\omega_{0}\right) t$ that is used to drive AOD2. The single sideband filtering can also be done optically by spatial filtering in the Fourier planes of AOD1 and AOD2. By evaluating Eq. (5) with the new signal values inserted, we can approximate the current generated by the $n$th detector-amplifier combination as

$$
i_{n}(t) \approx G \cos \left(2 \omega_{c} t-n \frac{\omega_{0} d}{M \nu_{a}}\right) .
$$

Note that in this case the antenna carrier frequency stays fixed at $2 \omega_{c}$ and is independent of the scan control frequency $\omega_{0}$. In other words, when the antenna beam is scanned by changing the control frequency $\omega_{0}$, the antenna carrier remains unchanged. This mode of antenna operation is suitable when the available antenna bandwidth is limited and can be used for transmitting information signals on a fixed carrier.

\section{Antenna Processor Optical Design and Experiments}

The AO beam steerer shown in Fig. 2 was demonstrated in the laboratory. This processor uses a pair of tellurium dioxide Bragg cells. The optics used in the processor consists of a $20-\mathrm{cm}$ collimating spherical lens, a $30-\mathrm{cm}$ focusing cylindrical lens for AOD $1,20-\mathrm{cm}$ spheres for 1:1 imaging, 25- and 30-cm spheres for imaging and Fourier plane filtering, and a $7.5-\mathrm{cm}$ spherical lens for the magnification system. The specifications of the Bragg cells were $70-\mu$ s aperture, $60-\mathrm{MHz}$ center frequency, and $30-\mathrm{MHz}$ bandwidth, giving 2100 resolvable deflection spots. The $N$-element high-speed detector array is simulated by using two detectors mounted on $x-y$ micrometer translation stages, allowing for the detector spacing $d$ to be adjusted according to design specifications, while placing the detectors at any position along the focused slit of light. The detector-amplifier set was a high-speed UDT PIN-HR008 photodiode with a 3-ns rise time, 200- $\mu \mathrm{m}$ diameter, $0.33-\mathrm{A} / \mathrm{W}$ responsivity at $514 \mathrm{~nm}$, and a high-speed UDT amplifier with 3-dB bandwidth of $500 \mathrm{kHz}$ to $400 \mathrm{MHz}$ and transimpedance gain of $3.5 \times$ $10^{3} \mathrm{~V} / \mathrm{A}$. As the Bragg cell acts as a beam deflector with a finite number of deflected spots, the control frequency is discretized and written as $f_{0}=p \delta f_{0}, p=0$, $1,2, \ldots$, and $\delta f_{0}=1 / T_{a}$, where $T_{a}$ is the illuminated Bragg cell aperture. The processor design equation can be written as

$$
\theta_{0}=\sin ^{-1}\left[(a / \lambda)\left(\frac{p \delta f_{0} d}{M \nu_{a}}-m\right)\right] \text {. }
$$

The design procedure for the processor is as follows: (a) Choose a value for $a / \lambda$ according to how far the grating lobes are to be placed in the array beam pattern. The analysis to follow uses $a / \lambda=0.5$, giving no grating lobes over a $\pm 90^{\circ}$ scan angle. (b) Choose the AOD to be used in the processor. This will determine the minimum value of control frequency resolution $\delta f_{0}$, the acoustic velocity $\nu_{a}$, and the maximum antenna carrier frequency possible. (c) Choose $\theta_{0 \min }$, the minimum scan angle required for the antenna corresponding to $p=1$. (d) Now solve Eq. (19) with $m=0$ to get the value for $M / d$ necessary to give the desired minimum scan angle for the smallest value of $f_{0}$. (e) Choose a tolerable phase-sampling error $\eta$ that is due to the finite size of the detector. Here $\eta=\left(d_{x} / d\right) \times 100$, where $d_{x}$ is the width of the detector's light-sensitive area along the phase-sampling direction. This gives the value for $d$, the detector spacing. Calculate the required optical magnification $M$, given the value of $d$. The processor design is complete.

The design for the experimental processor was chosen as $a / \lambda=0.5, \delta f_{0}=14.29 \mathrm{kHz}$, and $\nu_{a}=0.617 \mathrm{~mm} / \mu \mathrm{s}$ for $\mathrm{TeO}_{2}$ cells. $M=4.17$, the center frequency for the Bragg cell used, is $f_{c}=60 \mathrm{MHz}$, and an argon-ion laser (514-nm) light source was used. Table I shows the

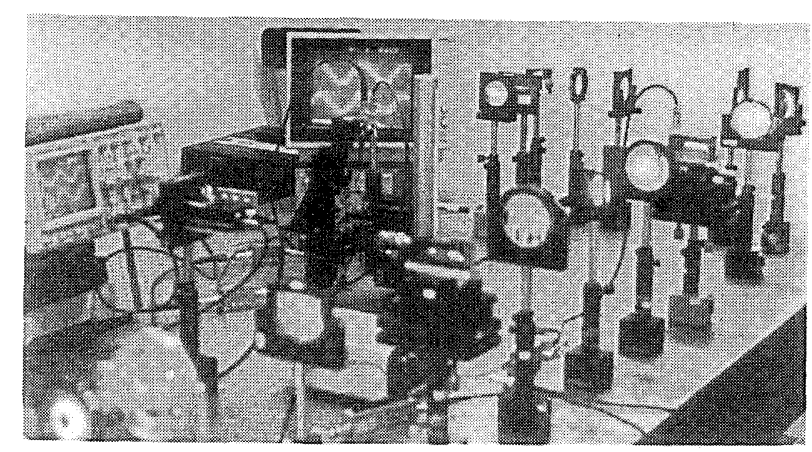

Fig. 5. Laboratory system.

Table I. System Design Values

\begin{tabular}{rcc}
\hline$d(\mathrm{~mm})$ & $\eta(\%)$ & $\theta_{0 \min }(\mathrm{deg})$ \\
\hline 5.9 & 3.4 & 3.8 \\
10.7 & 1.9 & 6.8 \\
\hline
\end{tabular}



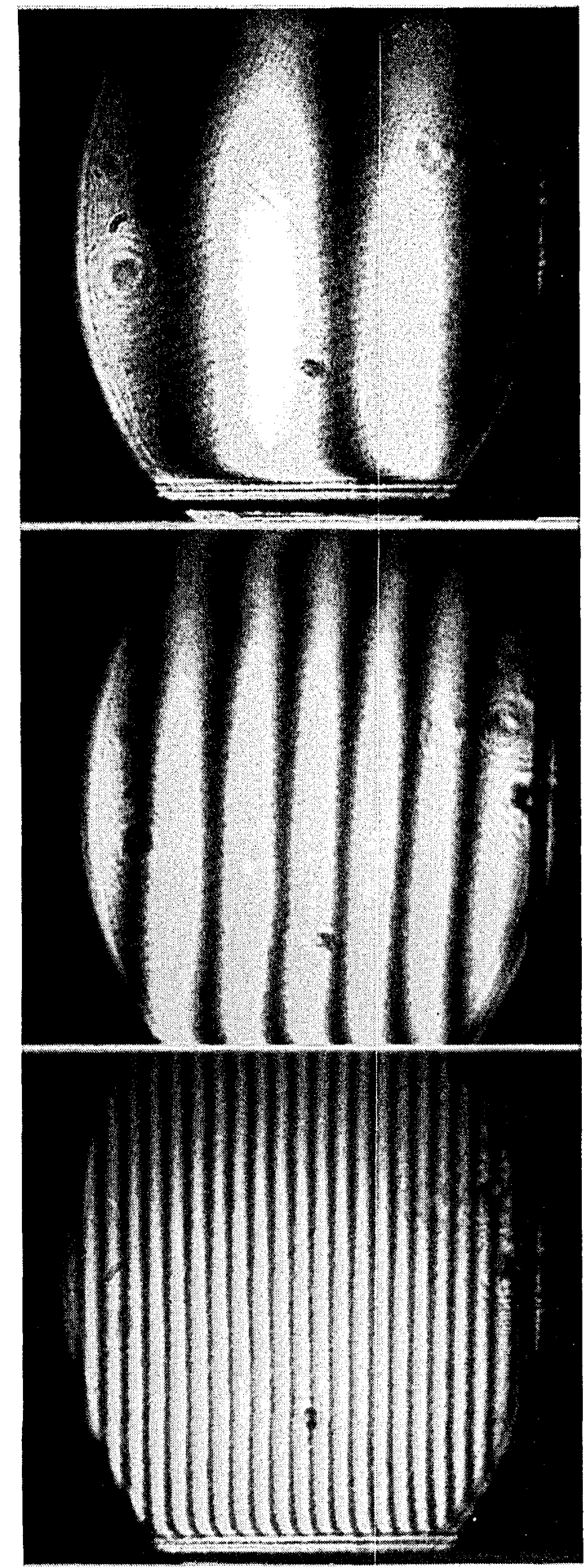

Fig. 6. Spatial-phase pattern sampled by the detectors shown with increasing control frequency (top to bottom).

other design parameters for the system. The $\mathrm{TeO}_{2}$ Bragg cell laboratory system is shown in Fig. 5; experimental results are shown in Figs. 6-8. To visualize the spatial-phase pattern that the detectors sample, we change the optical system such that we use the +1 orders from both of the Bragg cells, thus enabling us to detect a fringe pattern that is not on a temporal carrier.
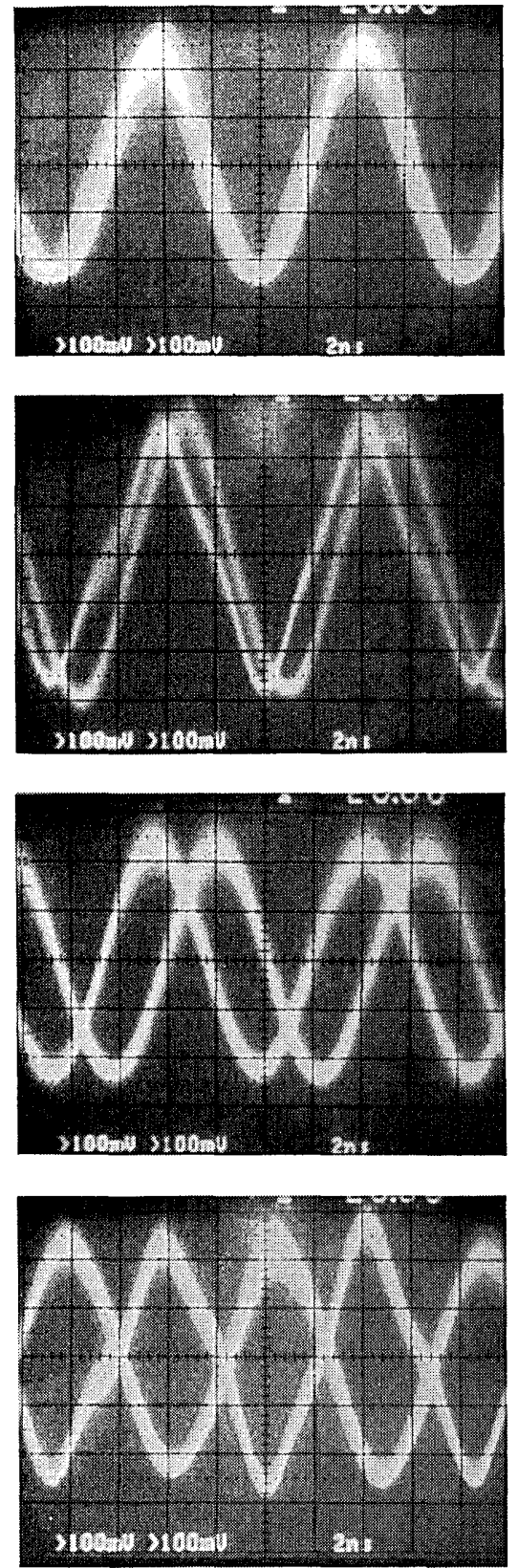

Fig. 7. Increasing phase difference between signals generated from the detector pair for increasing control frequency. $\Delta \psi=0, \pi / 4, \pi / 2$, $\pi$ (top to bottom).

This allows the charge-coupled device to pick up the spatial-phase pattern. Figure 6 shows this pattern for an increasing scan control frequency signal that is fed to both of the Bragg cells. Note the increase in spatial variation with increasing control frequency. This behavior becomes the basis for generating appropriately phased signals for the array antenna. Figure 7 shows the varying phase difference between the two signals generated from the detector pair positioned with a certain interdetector distance $d$. Here the control frequency is varied to obtain a $0-\pi$ phase shift for the generated carrier signals. Figure 8 shows the phase change for a fixed control frequency when the distance 

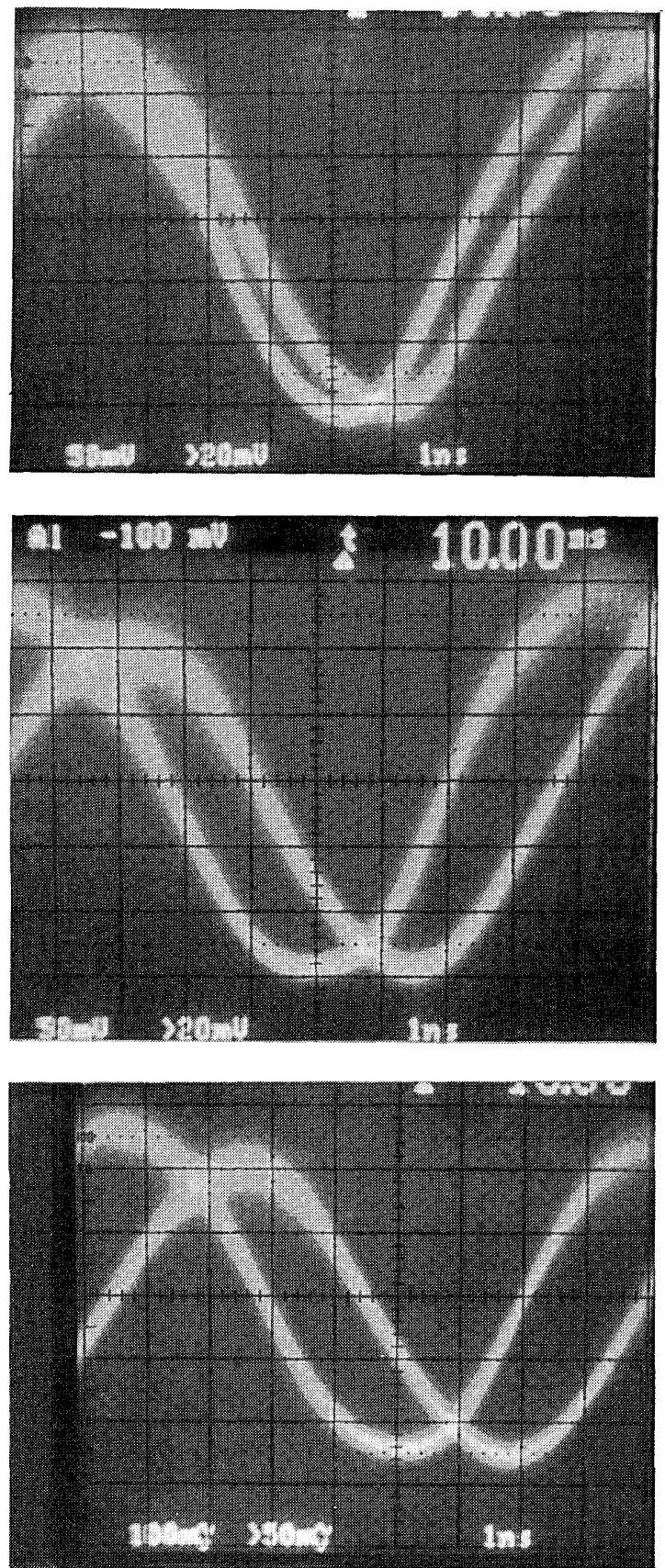

Fig. 8. Increasing phase difference between signals generated from the detector pair for an increasing (top to bottom) interdetector spacing.

between the detectors is increased, showing a gradually larger phase difference.

\section{Processor Performance Issues}

\section{A. Phase Linearity}

For the processor, the relationship between the detected signal phase change and the control frequency is linear. This rate of phase change with frequency can be expressed as

$$
s_{a}=\varphi / f_{0}=0.36 d / M \nu_{a}(\mathrm{deg} / \mathrm{kHz}) .
$$

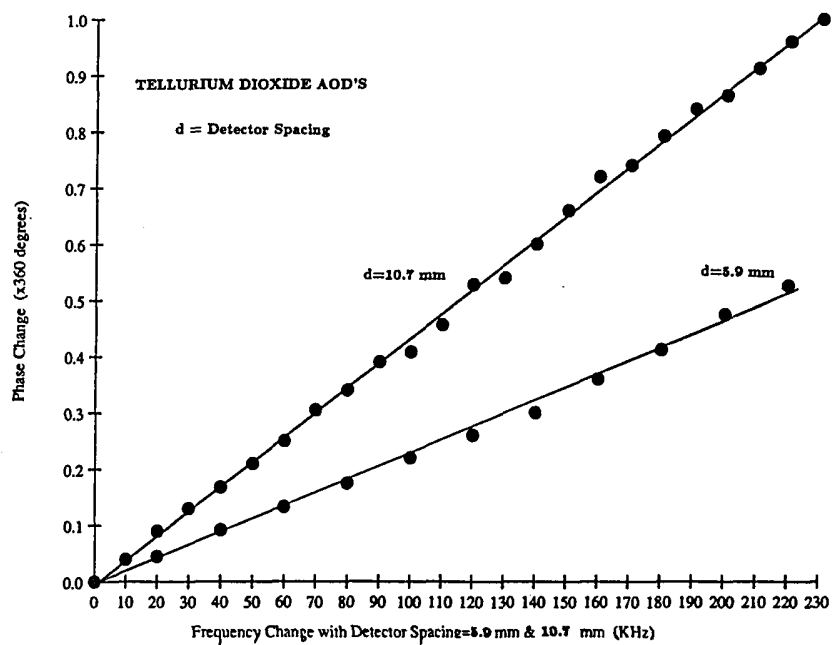

Fig. 9. Plot showing signal phase change versus control frequency.

Table II. Experimental Percentage Error Values

\begin{tabular}{rccl}
\hline$d(\mathrm{~mm})$ & $s_{a}(\mathrm{deg} / \mathrm{kHz})$ & $s_{e}(\mathrm{deg} / \mathrm{kHz})$ & $E_{s}(\%)$ \\
\hline 5.9 & 0.826 & 0.83 & 0.48 \\
10.7 & 1.5 & 1.512 & 0.8 \\
\hline
\end{tabular}

The percentage error is the rate

$$
E_{s}=\frac{\left|s_{a}-s_{e}\right|}{s_{a}} \times 100,
$$

where $s_{e}$ is the slope measured from the experimental data. As expected, the experimental plots for phase change with change in control frequency show a linear relationship (see Fig. 9). Note that when the detector spacing $d$ is doubled, the slope $s_{e}$ is also doubled. The error $E_{s}$ is calculated from the data and is very small for the different experimental setups (see Table II). This slope error is useful when one is calibrating the processor and will tell how far the processor is from its designed performance. Note that the signal phasechange data are measured by recording the signal time delay on the oscilloscope. Improved phase measurements could be achieved if phase-detector circuits and phase-locked loop electronics were employed.

\section{B. Beam-Scan Angle}

The phased-array antenna beam position is $\theta=\theta_{0}$ in Eq. (19), where $\theta_{0}=0^{\circ}$ corresponds to the beam at the broadside position and $p$ and $m$ are the control frequency and $2 \pi$ phase multiple indices, respectively. Using the processor design values for the different experimental conditions, we compute the expected beam-scan behavior; the measured data points give the experimental performance (see Fig. 10). Note that the experimental scan angle variation matches the analytically designed behavior closely. The minimum scan angle occurs when $p=1$, while the maximum scan angle is obtained from the condition $\sin \theta_{0} \leq 1$ for $p=0$, $1,2, \ldots$ These minimum and maximum scan angles are calculated for the processors with $m=0$. The results are shown in Table III. 


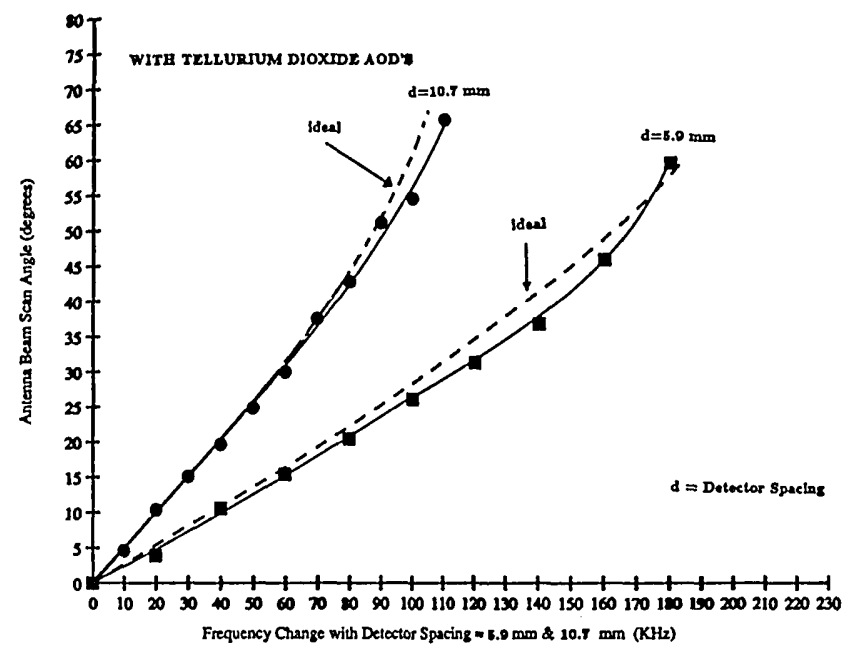

Fig. 10. Plot showing antenna beam-scan angle versus control frequency.

Table III. Designed Scan Angle Values

\begin{tabular}{rccc}
\hline$d(\mathrm{~mm})$ & $\theta_{0 \min }(\mathrm{deg})$ & $\theta_{0 \max }(\mathrm{deg})$ & $p_{\max }$ \\
\hline 5.9 & 3.8 & 80.75 & 15 \\
10.7 & 6.9 & 73.7 & 8 \\
\hline
\end{tabular}

\section{Number of Beam Positions}

The maximum number of beam positions for the phased-array antenna controlled by the optical processor is given by $N_{\text {beam }}=p_{\max }+1$, where $p_{\max }$ corresponds to the frequency index for the maximum scan angle. The +1 is added to include the broadside beam position. Note from Table III that an inverse relationship exists between the detector spacing and the number of independent beam positions for the phasedarray antenna. For example, in the experimental system with $d=5.9 \mathrm{~mm}$, there are 16 beam positions. When $\mathbf{d}$ is almost doubled to $10.7 \mathrm{~mm}$, the number of beam positions drops to 9 . Also note that the increase in beam positions is at the cost of increased control frequency bandwidth and higher detector phase-sampling error. The increased bandwidth of the carrier can be eliminated if we use the beam steerer 2 optical architecture.

\section{Detector Phase Sampling}

Ideally, the detector array acts as a spatial impulse train that samples phase values along the optically generated spatial carrier in the $x$ direction. In this section we analyze the effects of the finite size of the detector elements. The intensity incident upon the $n$th detector is given by

$$
\begin{aligned}
I_{n}(x, t)=\operatorname{bias}+G_{0} \cos \left(\omega t-\frac{\omega_{0}}{M \nu_{a}} x\right) & \operatorname{rect}\left(\frac{x-n d}{d_{x}}\right) \\
& \times \operatorname{rect}\left(\frac{y-0.5 d_{y}}{d_{y}}\right),
\end{aligned}
$$

where $d_{x}$ and $d_{y}$ are the width and the height of the detector, respectively. The signal generated after sig-
Table IV. Designed Values of the Product $u_{x} d_{x}$

\begin{tabular}{rc}
\hline$d(\mathrm{~mm})$ & $u_{x} d_{x}$ \\
\hline 5.9 & 0.0167 \\
10.7 & 0.0089 \\
\hline
\end{tabular}

nal integration of the incident light on the $n$th detector's photosensitive area is

$$
i_{n}(t)=\int_{0}^{d_{y}} \int_{-0.5 d_{x}+n d}^{0.5 d_{x}+n d} G_{0} \cos \left(\omega t-\frac{\omega_{0}}{M \nu_{a}} x\right) \mathrm{d} x \mathrm{~d} y+\text { bias. }
$$

Solving the integral, and substituting $u_{x}=f_{0} / M \nu_{a}$, we get

$$
\begin{aligned}
i_{n}(t) & =\frac{G_{0} d_{y}}{2 \pi u_{x}} \sin \left(\pi u_{x} d_{x}\right) \cos \left(\omega t-n 2 \pi u_{x} d\right)+\text { bias. } \\
& =\frac{G_{0} d_{x} d_{y}}{2} \operatorname{sinc}\left(u_{x} d_{x}\right) \cos \left(w t-n 2 \pi u_{x} d\right)+\text { bias. }
\end{aligned}
$$

Note that only the amplitude of the signal $i_{n}(t)$ is affected by the detector size, while the phase remains unaffected. Let us look at two cases.

If $u_{x}=0$, the current is

$$
i_{n}(t)=\frac{G_{0} d_{x} d_{y}}{2} \cos (\omega t)+\text { bias. }
$$

As expected, when there is no spatial carrier on the detector plane, only a temporal signal is generated without a phase term.

For the second case, if $u_{x} d_{x} \ll 1$, we can use the approximation $\sin \alpha \approx \alpha$ for small $\alpha$, giving the current

$$
i_{n}(t)=G \cos \left(\omega t-n 2 \pi u_{x} d\right)+\text { bias, }
$$

where $G=\left(G_{0} d_{x} d_{y}\right) / 2$. In other words, if the detector width is much smaller than the spatial carrier period, the detector samples over the small part of the fringe cycle that has approximately constant intensity. In this case, the amplitude apodizing sinc function can be approximately replaced by the constant 1 . This gives Eq. (26). Thus we can approximate the finite-size detector phase sampling as an impulse function sampling train, a result also physically obvious. The values of $u_{x} d_{x}$ for the experimental processor are calculated by using the detector width $d_{x}=200 \mu \mathrm{m}$ and the maximum value of the control frequency, that is, $p_{\max } \delta f_{0}$. Table IV shows the values. These data show that indeed the experimental designs satisfy the detector phase-sampling condition $u_{x} d_{x} \ll 1$. Note that, for systems using fibers for sampling, $d_{x}$ would be much smaller, that is, near $10-20 \mu \mathrm{m}$, resulting in a much smaller magnification $M$ for the imaging system.

\section{E. Beam-Switching Speed}

How fast we can switch a beam from one angular position to another depends on how quickly all the detectors provide steady-state correctly phased antenna drive signals. Although the proposed phased-array antenna optical processor has a parallel current feed format, the individual signal generation is based on a series-fed structure. Assuming that a length $X$ of the two respective AOD's is illuminated with Bragg- 
matched light beams, it takes $X / 2 \nu_{a} \mathrm{~s}$ for the two acoustic signals to cross and $X / \nu_{a}$ s for complete interaction between the two signals to occur. At this stage, all the detectors are illuminated by the acoustically modulated light signals, and phased currents are being generated for the radiating antennas. Thus the processor's frequency response depends on the Bragg cell's fill time, given by $T_{a}=X / \nu_{a} . T_{a}$ is also called the array fill time and affects the transient response of the antenna when the transmitted pulse width $T_{p}$ is of the order of the array fill time. A useful quantity is the antenna impulse response $h(t)$ since the antenna response at a target that is due to a signal $s(t)$ is the convolution of $h(t)$ with $s(t)$. The impulse response of the antenna-optical processor configuration is approximately given by ${ }^{12}$

$$
h(t, \theta)=\frac{M M_{1} X}{T_{a}} \operatorname{rect}\left(\frac{t}{T_{a}}\right) \exp \left[j 2 \pi\left(2 f_{c}+f_{0}\right) t\right],
$$

where $M_{1}$ is a system constant. This expression gives the maximum instantaneous 3-dB bandwidth of the processor to be $1 / 2 T_{a}$, which is also the maximum beam position switching rate. The analytical results show that indeed the beam-scanning speed is determined by the array (or AOD) fill time. Current AO devices have $T_{a}$ ranging from 100 to $0.2 \mu \mathrm{s}$, implying a considerably fast maximum beam switching-scanning speed of 2500 beams $/ \mathrm{ms}$. Note that there is a trade-off between the maximum number of beams and the beam-switching speed. In other words, for a fixed interdetector spacing, as we decrease the AOD optical aperture $X$ to increase the beam-switching speed, fewer detectors are illuminated by the diffracted optical beams. Since the maximum number of antenna beams possible is proportional to the number of active detectors in the system, the system with fewer active detectors generates fewer antenna beams. Nevertheless, one way of preserving the maximum number of beams from a system while keeping a fixed interdetector spacing is to use an optical magnification system that compensates for the reduction of the aperture $X$. In effect, the magnification system maintains illumination of all the detectors in the system when the optical aperture $X$ is reduced to increase the beamswitching speed.

\section{System Applications}

\section{A. Single- and Continuous-Mode Beam Scanning}

The optical processor can be used to position the phased-array antenna beam at discrete angular positions or to scan the antenna beam continuously over a wide angular region. For single-mode beam positioning, a specific control frequency is fed to the AOD to give the appropriate scan angle. For continuous beam scanning, a piecewise linear FM signal is fed to the AOD. In addition, multiple simultaneous arbitrary beams in space can be generated by a system similar to the single-beam generation system, except that the single laser source is replaced by a laser diode array
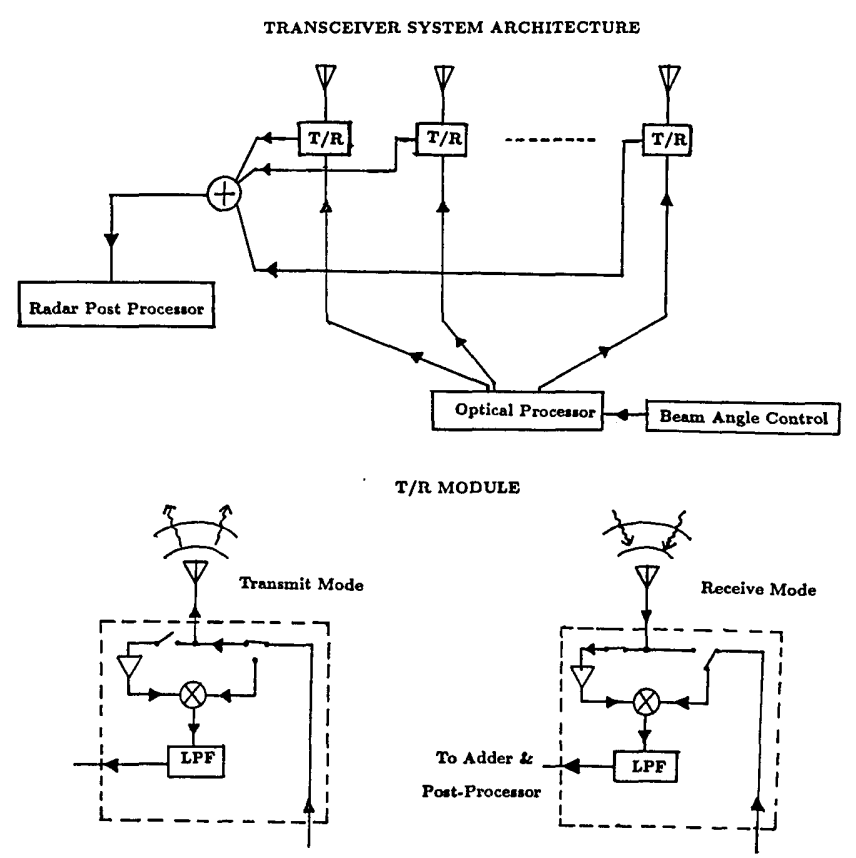

T/R MODULE

From Optical Driver

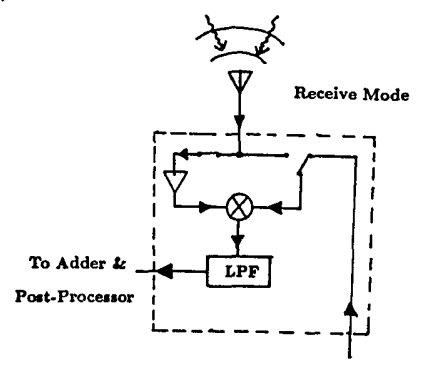

From Optical Drive

Fig. 11. Phased-array antenna transmission-receiving system.

and the second AOD is replaced by a multichannel AOD. ${ }^{12,13}$

\section{B. Antenna Receiving System}

The phased-array antenna receiving system is shown in Fig. 11, along with the transmitter part of the system. This receiving system discriminates the angle, range, and Doppler information of targets in the radar search range by processing target return signals from the array antenna elements. The basis of the postprocessing involves reusing the optically generated array transmit signals to cancel the phase factors associated with the received signals from the individual antenna elements, thus generating in-phase signals from all the individual returns. For target detection at a scan angle $\theta_{0}$ to broadside, we transmit a rf pulse $p_{T}(t)$ of duration $T$ on a carrier frequency $2 \omega_{c}+\omega_{0}$, with appropriate phase $\varphi_{0}$ set by the optical processor. The signal driving the $n$th antenna in the phased array is

$$
i_{n}(t)=p_{T}(t) G \cos \left[\left(2 \omega_{c}+\omega_{0}\right) t-n \varphi_{0}\right] .
$$

After transmitting the radar pulse, the antenna system is operated in the receive mode. The return signal at the $n$th antenna from a target at $\theta_{0}$ to broadside is approximately given by ${ }^{1}$

$$
i_{n}\left(t^{\prime}\right)=p_{T}\left(t^{\prime}\right) G_{T R}(n) \cos \left[\left(2 \omega_{c}+\omega_{0}+\omega_{d}\right) t^{\prime}-n \varphi_{0}\right],
$$

where

$$
\begin{aligned}
& p_{T}\left(t^{\prime}\right)=\operatorname{rect}\left(t^{\prime} / T\right), \quad t^{\prime}=t-\tau_{R}, \quad \tau_{R}=\text { range delay }=2 R / c \\
& \omega_{d}=\text { Doppler frequency }=-2 R \frac{\mathrm{d} R}{\mathrm{~d} t} \frac{\left(2 \omega_{c}+\omega_{0}\right)}{c}
\end{aligned}
$$


$R$ is the target range for a monostatic system, and $G_{T R}(n)$ is the signal gain dependent on target properties such as radar cross section. The signal in Eq. (29) is a time-delayed, frequency-shifted replica of the signal transmitted by the $n$th antenna in the array. Here we have assumed that the pulse width $T$ is much greater than the maximum interantenna element time delay, i.e., $T \gg N a / c$, and the Doppler frequency satisfies $\omega_{d} \ll 2 \omega_{c}+\omega_{0}$. The received signals are amplified and then mixed with their corresponding transmitter signals. The output of the mixer consists of a low-frequency Doppler signal, without the phase term $n \varphi_{0}$, and of a high-frequency signal. The high-frequency signal is filtered out by the low-pass filter, and the output of the $n$th $T / R$ module filter is approximately given by

$$
i_{n}\left(t^{\prime}\right) \approx p_{T}\left(t^{\prime}\right) G(n) \cos \left(\omega_{d} t^{\prime}\right) \text {. }
$$

Note that this signal has a $T$-second time duration with a $t=\tau_{R}$ time delay with respect to the reference transmit time of $t=0$. Also, all the signals from the different $T / R$ modules are in phase. Next, these in-phase signals are added up to maximize the receiver output signal-to-noise ratio, giving the signal

$$
i\left(t^{\prime}\right)=\sum_{n=0}^{N-1} i_{n}\left(t^{\prime}\right)=N G \cos \left(\omega_{d} t^{\prime}\right),
$$

where we have assumed constant amplitude $G$ for all the $T / R$ signals. This output signal contains the necessary range and Doppler (motion) information of the detected target at the preset scan angle $\theta_{0}$. This receiving system can also be used to form a simultaneous multiple-target tracking system, where simultaneous multiple beams in space are required in order to track a fast-moving target. ${ }^{12,13}$

\section{Features of the Optical Technique}

The optical and electronic designs of the system are relatively simple. The processor requires a single control parameter, namely, the frequency of a signal to control the antenna beam position in space. This control signal, being analog, allows for a gradual antenna response with changes in the control parameter. We no longer require the multiple control signals from the extensive computer hardware and software needed for controlling digital phase shifters. There is a direct parallel feed from the individual current drivers in the optical processor to the respective array antenna elements. Unlike the commonly used corporate feed structure that requires microwave power splitters for signal distribution, this direct parallel feed network is less susceptible to complete system failure, permitting a graceful degradation with component failure. Signal phase shift for the antennas is obtained from a global process of controlled optical beam interference and not from local phase-shifting devices. In this way we do away with the individual phase shifters and their support hardware required for the antenna elements.

Another feature of this optical technique is that the optical phase control mechanism (hardware) is inde- pendent of the antenna carrier frequency. This is unlike most conventional phase-shift mechanisms, in which a frequency-dependent material parameter is used to introduce the phase shift. This frequency independence feature of the optical technique gives an antenna operator greater freedom to switch among different carrier frequencies. The Doppler effect in the two AOD's in the optical processor doubles the input AOD drive frequency. This feature permits the use of a signal source of half of the desired antenna carrier frequency. Thus, since the oscillator is lower in frequency (e.g., 2 instead of $4 \mathrm{GHz}$ ), it is more stable, less expensive, and easier to maintain than a higherfrequency signal generator. Another feature is that a linear phased array can easily be changed from a uniform linear array to a nonuniform linear array by simply rearranging the phase-sampling detectors (fibers) in the desired format. This readily changes the transmit-antenna radiation pattern. The optics for the proposed processor is highly stable since the system is an in-line additive system. In other words, the two interfering beams in the system travel through almost identical paths, and any system imperfections affect both beams equally and simultaneously. Finally, optical signals are less susceptible to electromagnetic interference effects, particularly when fibers are used to carry the phased-array signals to the antenna elements to reduce further the internally generated electromagnetic fields that could affect the surrounding antenna system components. ${ }^{7}$

\section{Extensions}

The proposed optical processor has the potential of being a highly engineered, compact processing unit, built with existing bulk (discrete) devices or in an integrated form on a suitable substrate. Figure 12 shows a compact design of the optical processor using

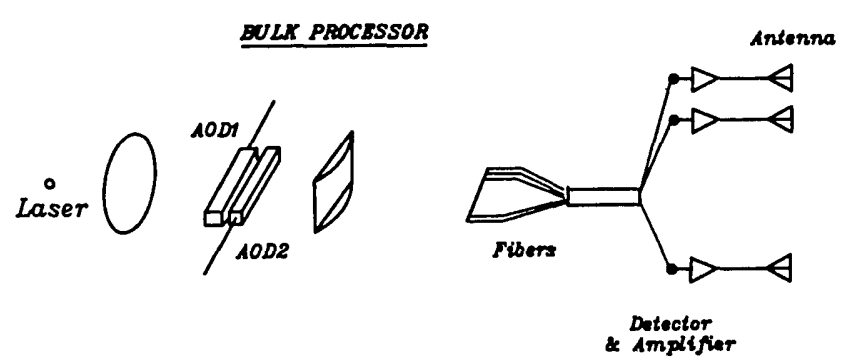

Fig. 12. Compact design of the optical processor.

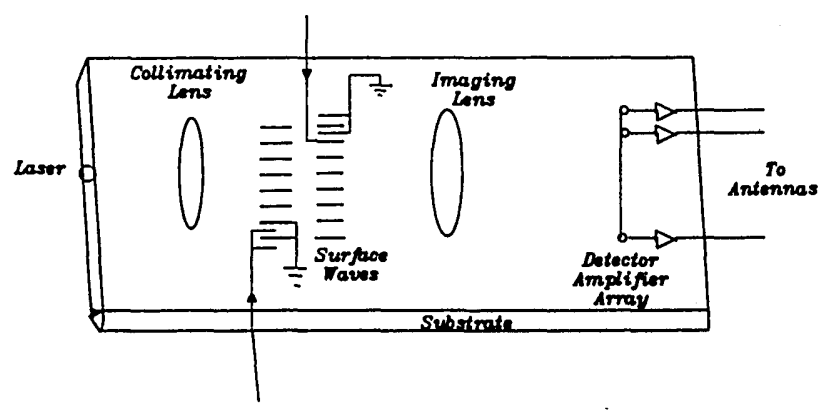

Fig. 13. Integrated optic version of the processor. 
bulk AO devices, while Fig. 13 shows an integrated optical version of the optical processor using surface acoustic wave devices made on a suitable substrate such as $\mathrm{LiNbO}_{3}$. The integrated optical antenna processor can be preferable for airborne and spaceborne systems. In this processor, the maximum antenna carrier frequency is limited by the highest AOD transducer center frequency available. Current practical limits on the center frequency are in the $3-\mathrm{GHz}$ range, giving an antenna carrier of $6 \mathrm{GHz}$. This permits operation up to the C-band radar range. The onedimensional optical linear phased-array processor can be modified to provide several applications, such as multiple linear array feeds, nonuniform phase sampling to generate various antenna patterns, single-frequency beam azimuth-height control, and independent beam azimuth-height control by means of two control signals. ${ }^{12,13}$

\section{Conclusion}

In this paper we have introduced simple, compact, and powerful phase-based $\mathrm{AO}$ techniques for control and signal processing in phased-array antennas. The proposed optical approach eliminates the need for the phase shifters required in typical electronically controlled phased arrays. Using a single control signal, this technique simultaneously provides the correctly phased signals for transmission and reception. The principles of the optical phased-array antenna processor for the transmission mode have been experimentally demonstrated. The results from the processor are in close agreement with the analytical design data. In addition, there is the possibility of integrating the processor on a substrate, thus leading to smaller and lighter phased-array antenna systems. Further research relates to fabricating a prototype of this system and studying the extensions of this processor. ${ }^{13}$

This research was performed at the California Institute of Technology in conjunction with Nabeel A. Riza's Ph.D. dissertation. It was supported, in part, by the U.S. Air Force Office of Scientific Research.

\section{References}

1. L. B. Lambert, M. Arm, and A. Aimette, "Electro-optical signal processors for phased array antennas," in Optical and ElectroOptical Information Processing, J. T. Tippett, D. A. Berkowitz,
L. C. Clapp, C. J. Koester, and A. Vanderburgh, Jr., eds. (MIT Press, Cambridge, Mass., 1965), Chap. 38.

2. M. King, W. R. Bennett, L. B. Lambert, and M. Arm, "Real-time electro-optical signal processors with coherent detection," Appl. Opt. 6, 1367-1375 (1967).

3. D. Casasent and F. Cassasayas, "Electro-optical processing of phased array antenna data," IEEE Trans. Aerosp. Electron. Syst. AES-11, 65 (1975).

4. D. Casasent, "Radar signal processing," in Optical Data Processing: Applications, D. Casacent, ed., Vol. 23 of Topics in Applied Physics (Springer-Verlag, Berlin, 1977), Sec. 8.4.

5. K. B. Bhasin and B. M. Hendrickson, eds., Opto-Electronic Signal Processing for Phased Array Antennas, Proc. Soc. Photo-Opt. Instrum. Eng. 886 (1988).

6. J. J. Pan, "Fiber optics for wideband extra high frequency (EHF) phased arrays," in Opto-electronic Signal Processing for Phased Array Antennas, K. B. Bhasin and B. M. Hendrickson, eds., Proc. Soc. Photo-Opt. Instrum. Eng. 886, 60-70 (1988).

7. M. H. Popek, "Electro-optic system components for phased array radar applications," in Opto-Electronic Signal Processing for Phased Array Antennas, K. B. Bhasin and B. M. Hendrickson, eds., Proc. Soc. Photo-Opt. Instrum. Eng. 886, 32-45 (1988).

8. G. A. Koepf, "Optical processor for phased array antenna beam forming," in Optical Technology for Microwave Applications, S.-K. Yao, ed., Proc. Soc. Photo-Opt. Instrum. Eng. 477, 75-81 (1984).

9. L. P. Anderson, F. Boldissar, and D. C. D. Chang, "Antenna beamforming using optical processing," in Opto-Electronic Signal Processing for Phased Array Antennas, K. B. Bhasin and B. M. Hendrickson, eds., Proc. Soc. Photo-Opt. Instrum. Eng. 886 (1988), paper 27.

10. I. C. Chang, and S. S. Tarng, "Phased array beamforming using acousto-optic techniques," in Advances in Optical Information Processing III, D. R. Pape, ed., Proc. Soc. Photo-Opt. Instrum. Eng. 936, 163-167 (1988).

11. N. A. Riza and D. Psaltis, "An acousto-optic technique for beam scanning and beam formation in phased array radars," in Annual Meeting Technical Digest, Vol. 11 of OSA Technical Digest Series (Optical Society of America, Washington, D.C., 1988), paper ThT5.

12. N. A. Riza, "Novel acousto-optic systems for spectrum analysis and phased array radar signal processing," $\mathrm{Ph} . \mathrm{D}$. dissertation (California Institute of Technology, Pasadena, Calif., 1989).

13. N. A. Riza, "Acousto-optic architectures for multidimensional phased array antenna processing," in Optical Technology for Microwave Applications V, S. Yao, ed., Proc. Soc. Photo-Opt. Instrum. Eng. 1476, (to be published).

14. M. L. Skolnik, Introduction to Radar Systems (McGraw-Hill, New York, 1981), Chap. 8.

15. R. J. Mailloux, "Phased array theory and technology," Proc. IEEE 70, 246-291 (1982). 